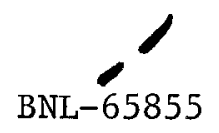

\title{
Marcus equation
}

In the late 1950s to early 1960s Rudolph A. Marcus developed a theory for treating the rates of outer-sphere electron-transfer reactions. Outer-sphere reactions are reactions in which an electron is transferred from a donor to an acceptor without any chemical bonds being made or broken. (Electron-transfer reactions in which bonds are made or broken are referred to as inner-sphere reactions.) Marcus derived several very useful expressions, one of which has come to be known as the Marcus cross-relation or, more simply, as the Marcus equation. It is widely used for correlating and predicting electron-transfer rates. For his contributions to the understanding of electron-transfer reactions, Marcus received the 1992 Nobel Prize in Chemistry.

In common with ordinary chemical reactions, an electron-transfer reaction can be described in terms of the motion of the system on an energy surface. As the reaction proceeds the system moves from the reactant minimum (initial state) to the product minimum (final state). The nuclear configurations of the reactants and products and the configuration of the surrounding solvent are constantly changing as a consequence of thermal motion. Marcus showed that, subject to certain assumptions, these fluctuations can be described in terms of displacements on harmonic free-energy curves that are a function of a single reaction coordinate. Two harmonic free-energy curves are needed to describe the reaction - one refers to the reactants plus surrounding medium and the other to the products plus surrounding medium. The two free-energy curves have identical force constants and the reaction coordinate is the difference between the reactant and product free energies at a particular nuclear configuration.

The free energy of the close-contact reactants plus surrounding medium (Curve R) and the free energy of the close-contact products plus surrounding medium (Curve P) are plotted vs. the reaction coordinate in Figure 1. The plot is for an electron-transfer reaction with zero standard free-energy change (an electron self-exchange reaction). The free-energy curves intersect where the reactants plus surrounding solvent and the products plus surrounding solvent have the same nuclear configurations and energies. This intersection defines the transition state for the reaction: the energy required to reach the intersection is the free energy of activation for the reaction. Also shown is $\lambda$, the reorganization parameter, which is the vertical difference between the free energies of the (noninteracting) reactants and products of a self-exchange reaction at the 


\section{DISCLAIMER}

This report was prepared as an account of work sponsored by an agency of the United States Government. Neither the United States Government nor any agency Thereof, nor any of their employees, makes any warranty, express or implied, or assumes any legal liability or responsibility for the accuracy, completeness, or usefulness of any information, apparatus, product, or process disclosed, or represents that its use would not infringe privately owned rights. Reference herein to any specific commercial product, process, or service by trade name, trademark, manufacturer, or otherwise does not necessarily constitute or imply its endorsement, recommendation, or favoring by the United States Government or any agency thereof. The views and opinions of authors expressed herein do not necessarily state or reflect those of the United States Government or any agency thereof. 


\section{DISCLAIMER}

Portions of this document may be illegible in electronic image products. Images are produced from the best available original document. 
reactants' equilibrium configuration. Electronic interaction of the reactants gives rise to the splitting of the energy curves (noncrossing) at their intersection. This splitting is equal to $2 H_{\mathrm{ab}}$, where $H_{\mathrm{ab}}$ is the electronic coupling matrix element. The coupling of the reactants is assumed large enough so that the electron transfer occurs with unit probability at the transition state configuration but not large enough to lower the barrier significantly. The splitting is usually neglected in calculating the free energy of activation for the electron transfer.

Self-exchange reactions. The reactants and products of an electron selfexchange reaction are identical. An example is the $\mathrm{Fe}(\mathrm{phen}) 3^{2+}-\mathrm{Fe}(\mathrm{phen}) 3^{3+}$ selfexchange reaction shown in Eq. (1), where phen $=1,10$-phenanthroline.

$$
\mathrm{Fe}(\text { phen })_{3}^{2+}+\mathrm{Fe}(\text { phen })_{3}^{3+}=\mathrm{Fe}(\text { phen })_{3}^{3+}+\mathrm{Fe}(\text { phen })_{3}^{2+}
$$

In terms of the Marcus formalism, the rate constant for a self-exchange reaction is given by Eqs. (2) and (3)

$$
\begin{aligned}
& k=A \exp \left(-\Delta G^{*} / R T\right) \\
& \Delta G^{*}=\lambda / 4
\end{aligned}
$$

where $A$ is the collision frequency of the reactants in solution, $\Delta G^{*}$ is the activation free energy for the electron transfer with the two reactants in contact, and $\lambda$ is the reorganization parameter referred to above. The reorganization parameter or intrinsic barrier is comprised of solvational and intramolecular (vibrational) components. The solvational contribution depends upon the solvent dielectric properties, on the distance separating the donor and acceptor sites and, for a given separation, on the shape of the reactants. The intramolecular contribution depends upon the bond length changes and force constants and is generally treated within an harmonic approximation.

Net electron-transfer reactions. The oxidation of $\mathrm{Ru}\left(\mathrm{NH}_{3}\right)_{6}{ }^{2+}$ by $\mathrm{Fe}(\mathrm{phen}) 3^{3+}$, Eq. (4), is an example of an electron-transfer reaction accompanied by a net chemical change. Most electron-transfer reactions fall into this category. Because the reactants and products of a net reaction differ, there is a net freeenergy change in the reaction.

$$
\mathrm{Ru}\left(\mathrm{NH}_{3}\right)_{6}{ }^{2+}+\mathrm{Fe}(\text { phen })_{3}{ }^{3+}=\mathrm{Ru}\left(\mathrm{NH}_{3}\right)_{6}{ }^{3+}+\mathrm{Fe}(\text { phen })_{3}{ }^{2+}
$$


The free-energy curves for the reactants and products of an exergonic electrontransfer reaction, that is, a reaction accompanied by a decrease in free energy, are illustrated in Figure 2. The free energy of activation for such a reaction is given by Eq. (5)

$$
\Delta G^{*}=\left(\lambda+\Delta G^{\mathrm{o}}\right)^{2} / 4 \lambda
$$

where $\Delta G^{\circ}$ is the the standard free-energy change for the reaction with the two reactants in contact. Equation (5) reduces to the expression for the free energy of activation for a self-exchange reaction, Eq. (3), when $\Delta G^{\mathrm{o}}=0 .\left|\Delta G^{\mathrm{o}}\right|$ may be viewed as the the driving force for an exergonic electron-transfer reaction.

Three free-energy regimes can be distinguished depending on the relative magnitudes of the reorganization parameter and the driving force. When $\mid \Delta G$ o $\mid<$ $\lambda$ the reaction is in the normal regime where $\Delta G^{*}$ decreases, and the rate constant increases, with increasing driving force. This is the case for the reaction illustrated in Figure 2. The reaction becomes barrierless $\left(\Delta G^{*}=0\right)$ when $\left|\Delta G^{\circ}\right|=\lambda$. If the driving force is increased even further then $\mid \Delta G$ 이 $>\lambda$ and $\Delta G^{*}$ increases, and the rate constant decreases, with increasing driving force. This is the nonintuitive inverted regime.

Marcus cross-relation. Marcus showed that the rate constant $k_{12}$ for an electron-transfer reaction accompanied by a net chemical change is related to the rate constants $k_{11}$ and $k_{22}$ for the component self exchanges by

$$
\begin{gathered}
k_{12}=\left(k_{11} k_{22} K_{12} f_{12}\right)^{1 / 2} W_{12} \\
\ln f_{12}=\frac{\left[\ln K_{12}+\left(w_{12}-w_{21}\right) / R T\right]^{2}}{4\left[\ln \left(\frac{k_{11} k_{22}}{Z^{2}}\right)+\frac{w_{11}+w_{22}}{R T}\right]} \\
W_{12}=\exp \left[-\left(w_{12}+w_{21}-w_{11}-w_{22}\right) / 2 R T\right]
\end{gathered}
$$

where $K_{12}$ is the equilibrium constant for the cross-reaction, $w_{\mathrm{ij}}$ is the electrostatic work required to bring together the reactants (products) $\mathrm{i}$ and $\mathrm{j}$, and $Z$ is the collision frequency of two uncharged molecules in solution $\left(10^{11} \mathrm{M}^{-1} \mathrm{~s}^{-1}\right)$.

Equation (6) is based upon the assumption that the reorganization parameter for a 
cross-reaction is equal to the average of the reorganization parameters for the component self-exchange reactions, Eq. (7).

$$
\lambda_{12}=\left(\lambda_{11}+\lambda_{22}\right) / 2
$$

For example, if the oxidation of $\mathrm{Ru}\left(\mathrm{NH}_{3}\right)_{6}{ }^{2+}$ by $\mathrm{Fe}(\text { phen })_{3}{ }^{3+}$, Eq. (4), is the crossreaction $\left(k_{12}, \lambda_{12}\right)$, then the $\mathrm{Ru}\left(\mathrm{NH}_{3}\right)_{6}{ }^{2+}-\mathrm{Ru}\left(\mathrm{NH}_{3}\right)_{6}{ }^{3+}$ and $\mathrm{Fe}(\mathrm{phen})_{3}{ }^{2+}$ $\mathrm{Fe}$ (phen) $3^{3+}$ reactions, Eqs. (8) and (4), respectively, are the component selfexchange reactions $\left(k_{11}, \lambda_{11}\right.$ and $\left.k_{22}, \lambda_{22}\right)$.

$$
\mathrm{Ru}\left(\mathrm{NH}_{3}\right)_{6}{ }^{2+}+\mathrm{Ru}\left(\mathrm{NH}_{3}\right)_{6}{ }^{3+}=\mathrm{Ru}\left(\mathrm{NH}_{3}\right)_{6}^{3+}+\mathrm{Ru}\left(\mathrm{NH}_{3}\right)_{6}{ }^{2+}
$$

When the driving force is not too large and the work terms cancel then $\ln f_{12} \approx 0$ and $W_{12}=1$. Under these conditions Eq. (6) reduces to Eq. (9).

$$
k_{12}=\left(k_{11} k_{22} K_{12}\right)^{1 / 2}
$$

Equation (9) is often referred to as the Marcus equation. It has been extensively applied to electron-transfer reactions involving metal complexes. The stable oxidation states of transition-metal complexes generally differ by one electron and the metal centers and surrounding ligands can be independently varied to encompass a wide range of electron-transfer rates and driving forces. For moderately exergonic electron-transfer reactions between similarly charged complexes the agreement of the observed rates with those calculated from Eq. (9) is often remarkably good. At high exergonicities and/or when the net electron transfer involves oppositely charged complexes it is necessary to use Eq. (6). The rates of highly exergonic reactions calculated with the extended equation are almost always somewhat higher than the observed rates in the normal freeenergy regime and are slower than the observed rates in the inverted regime. Reasons advanced for the failure of the cross-relation include changes in reaction mechanism, anharmonicity contributions, nuclear tunneling contributions, and noncancellation of nonelectrostatic contributions to the work terms.

Examples of the application of the Marcus equation to organic and/or organometallic systems include the oxidation of tetraalkyltin, tetraalkyllead and dialkylmercury by $\mathrm{Fe}$ (phen) ${ }_{3}^{3+}$, the oxidation of various methylarenes, $\mathrm{ArCH}_{3}$, by substituted $\mathrm{Fe}\left(\right.$ phen) $3^{3+}$ complexes, Eq. (10), 


$$
\mathrm{ArCH}_{3}+\mathrm{Fe}(\text { phen })_{3}^{3+}=\mathrm{ArCH}_{3}{ }^{+}+\mathrm{Fe}(\text { phen })_{3}{ }^{2+}
$$

and the oxidation of $\mathrm{N}$-alkylpyridinyl radicals by $[\operatorname{Ir}(1,5$-cyclooctadiene $)(\mu$ pyrazolyl) $]_{2}{ }^{+}$, Eq. (11), where very good agreement with the predictions for highly exergonic electron transfer is obtained.

$$
\mathrm{C}_{6} \mathrm{H}_{5} \mathrm{~N}-\mathrm{R} \cdot+\mathrm{Ir}_{2}{ }^{+}=\mathrm{C}_{6} \mathrm{H}_{5} \mathrm{~N}-\mathrm{R}^{+}+\mathrm{Ir}_{2}
$$

The Marcus equation has also been succesfully applied to reactions of alkylhydrazines, ferrocene derivatives and $p$-phenylenediamine derivatives.

Because the reorganization barrier is an intrinsic property of a redox couple and independent of its reaction partner, a simple relationship should exist between the homogeneous self-exchange rate constant of a redox couple and its exchange rate constant at an electrode. The relationship between homogeneous and heterogeneous electron-transfer rate constants predicted by the Marcus formalism also holds up well in practice.

Proton, hydride, atom and group transfers. Although originally derived for electron-transfer reactions, the Marcus formalism can also be applied to reactions in which bond-making and bond-breaking occur. If the net reaction involves transfer of a covalently attached $X$ from a donor to an acceptor, then the cross-reaction and self-exchanges need to be redefined in terms of X-transfer reactions as shown in Eq. (12).

$$
\begin{aligned}
& A X+B=A+X B \\
& A X+A=A+X A \\
& B X+B=B+X B
\end{aligned}
$$

For example, the oxidation of a methylarene, Eq. (10), is followed by proton transfer from the methylarene cation radical to a substituted pyridine, Eq (13).

$$
\mathrm{ArCH}_{3} \cdot++\mathrm{Y}-\mathrm{py}=\mathrm{ArCH}_{2} \cdot+\mathrm{Y}-\mathrm{pyH}^{+}
$$

In this case $\mathrm{X}=\mathrm{H}^{+}, \mathrm{A}=\mathrm{ArCH}_{2} \cdot$, and $\mathrm{B}=\mathrm{Y}$-py. The Marcus formalism yields a consistent value for the intrinsic barrier for the proton transfer. Other 
applications include hydride transfers, Eq. (14), where $A_{i} N^{+} R$ is a nitrogen heterocycle, gas-phase atom transfers, Eq. (15), and nucleophilic displacements (methyl transfers), Eq. (16).

$$
\begin{aligned}
\mathrm{A}_{1}(\mathrm{H}) \mathrm{NR}+\mathrm{A}_{2} \mathrm{~N}^{+} \mathrm{R} & =\mathrm{A}_{1} \mathrm{~N}^{+} \mathrm{R}+\mathrm{A}_{2}(\mathrm{H}) \mathrm{NR} \\
\mathrm{RH}+\mathrm{CH}_{3} & =\mathrm{R}+\mathrm{CH}_{4} \\
\mathrm{ClCH}_{3}+\mathrm{F}^{-} & =\mathrm{Cl}^{-}+\mathrm{CH}_{3} \mathrm{~F}
\end{aligned}
$$

Since the transfer of the heavier particles generally involves strong electronic interactions, the $\mathrm{X}$ transfers are not well described by intersecting harmonic freeenergy curves. Use of a bond-energy bond-order (BEBO) model for the $\mathrm{X}$ transfers results in a free-energy expression similar to Eq. (5) when $\mid \Delta G$ ol $/ \lambda$ is small, but differs from it in not predicting an inverted regime at large $\left|\Delta G^{\mathrm{o}}\right| / \lambda$. The $\lambda$ parameter is found to have the same property as Eq. (5) and a cross-relation analogous to Eq.(6) is obtained.

The expression for the free-energy of activation, Eq. (5), is applicable to reactions that can be described in terms of two weakly interacting harmonic freeenergy curves. It does not apply when there is an appreciable contribution from bond making or rupture in the rate-determining step. The barrier averaging relation, Eq. (7), is, however, more general: intrinsic barrier additivity obtains in both electron and heavier particle transfers. Marcus' insight into the factors determining reaction rates, particularly the additivity of intrinsic barriers, has proved invaluable in understanding and predicting the rates of a variety of electron-, atom- and group-transfer reactions.

\section{Norman Sutin}

Bibliography. R. A. Marcus, Ann. Rev. Phys. Chem., Vol. 15, p. 155, 1964; N. Sutin, Prog. Inorg. Chem., Vol. 30, p. 441, 1983; R. A. Marcus and N. Sutin, Biochim. Biophys. Acta, Vol. 811, p. 265, 1985; L. Eberson, Electron Transfer Reactions in Organic Chemistry, 1987; R. A. Marcus, Les Prix Nobel, 1993; D. Astruc, Electron Transfer and Radical Processes in Transition-Metal Chemistry, 1995; R. A. Marcus, Pure \& Appl. Chem., Vol. 69, p. 13, 1997; S. F. Nelsen et al., J. Am. Chem. Soc., Vol. 119, p. 5900, 1997.

This research was carried out at Brookhaven National Laboratory under contract DE-AC02-98CH10886 with the U. S. Department of Energy and supported by its Division of Chemical Sciences, Office of Basic Energy Sciences. 
(Figure captions)

Figure 1. Free-energy curves for the close-contact reactants plus surrounding medium (left hand curve $\mathrm{R}$ ) and close-contact products plus surrounding medium (right hand curve $P$ ) of an electron self-exchange reaction. Also shown are the the reorganization parameter $\lambda$ and the splitting $2 H_{\mathrm{ab}}$ at the intersection of the (noninteracting) reactant and product curves.

Figure 2. Free-energy curves for the close-contact reactants plus surrounding medium (left hand curve $R$ ) and close-contact products plus surrounding medium (right hand curve $\mathrm{P}$ ) of an electron-transfer reaction accompanied by a standard free-energy change $\Delta G^{\circ}$. 


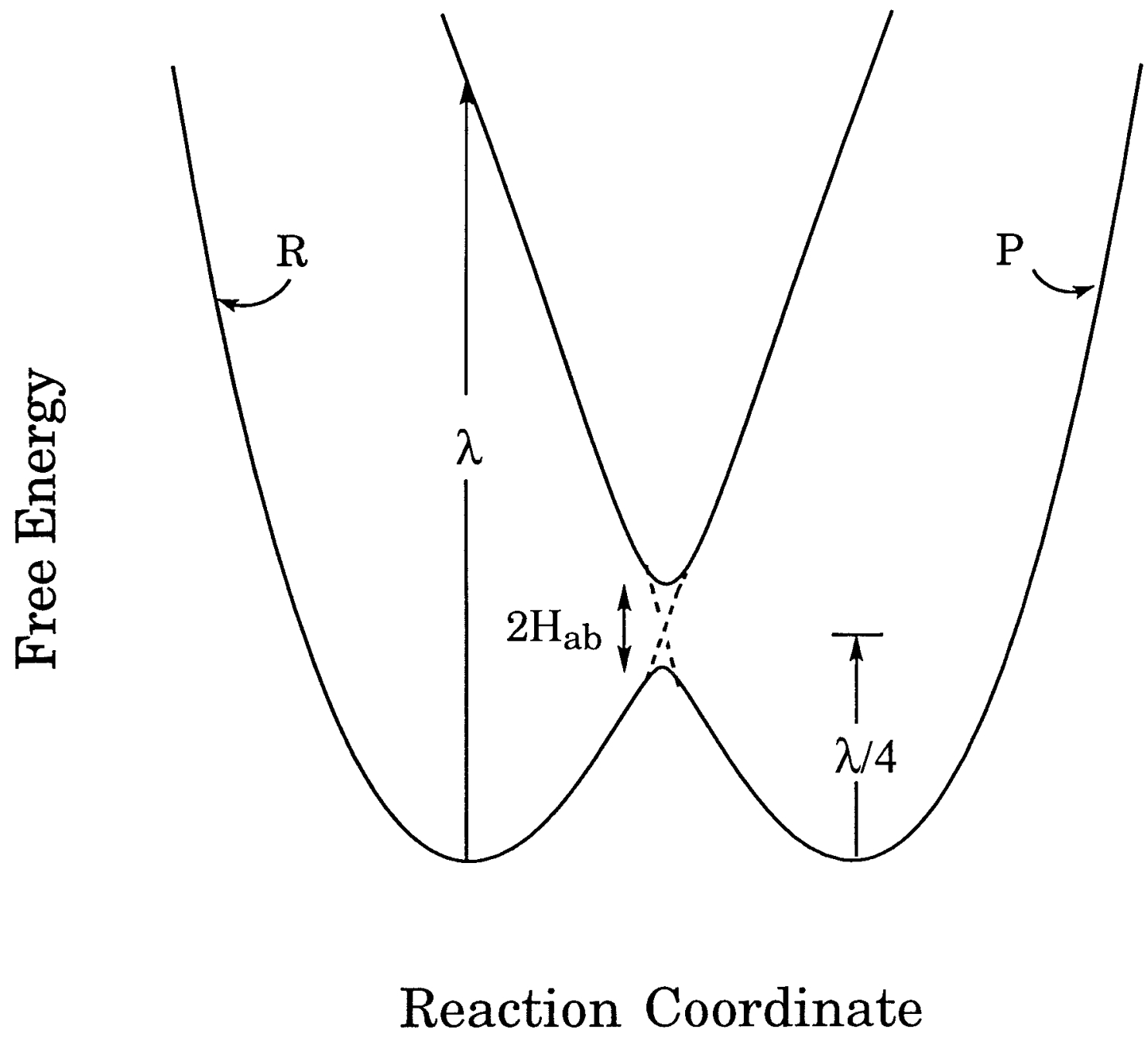




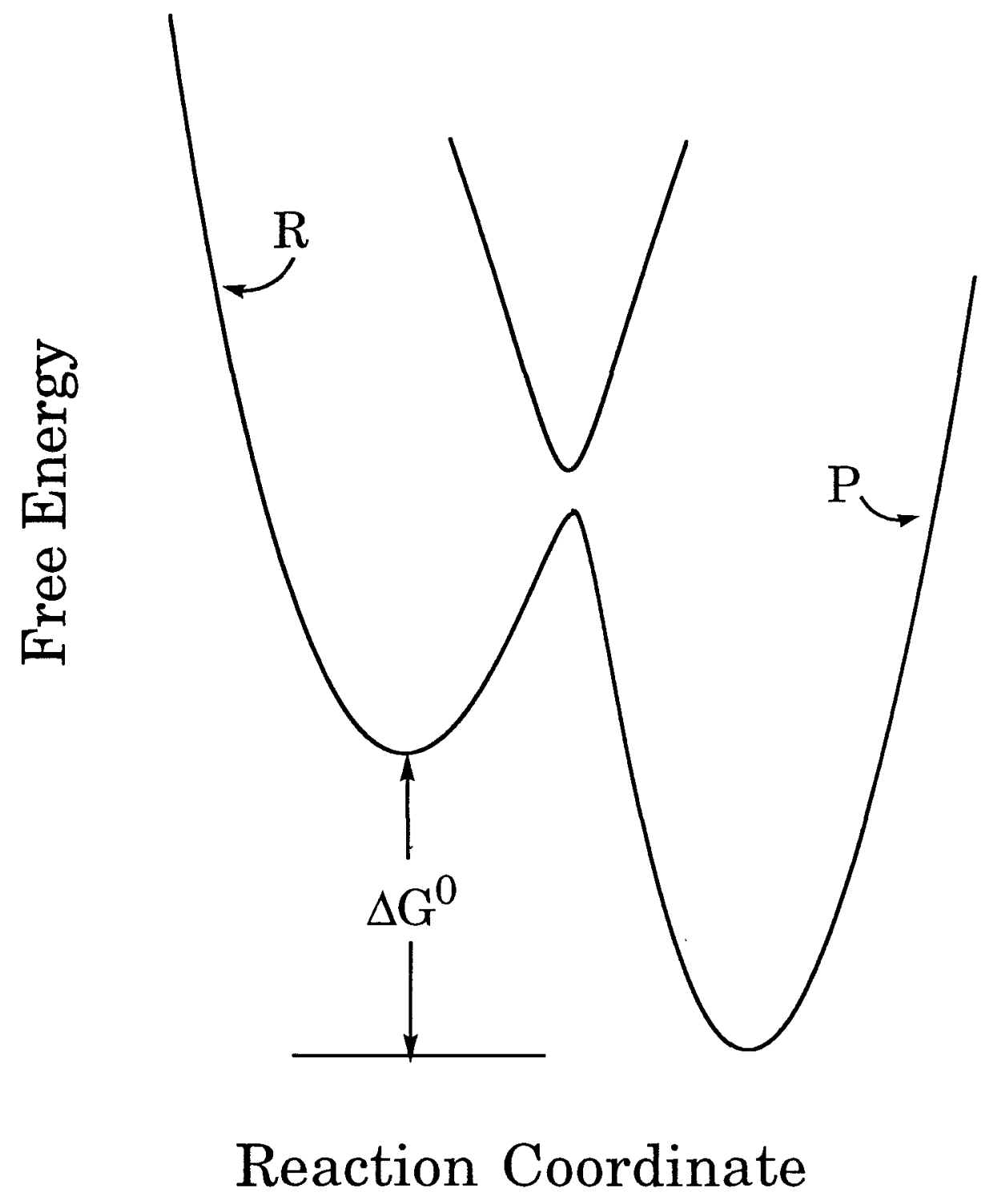

